\title{
Forecast of Outpatient Visits to a Tertiary Eyecare Network in India Using the EyeSmart Electronic Medical Record System
}

\author{
Gumpili Sai Prashanthi ${ }^{1,2} \oplus$, Nareen Molugu ${ }^{3}$, Priyanka Kammari ${ }^{1}$, Ranganath Vadapalli ${ }^{1}$ \\ and Anthony Vipin Das $1,2, *$ (D) \\ 1 Department of EyeSmart EMR \& AEye, L. V. Prasad Eye Institute, Hyderabad 500034, India; \\ saiprashanthi.g@lvpei.org (G.S.P.); priyankakammari19@gmail.com (P.K.); ranganath@lvpei.org (R.V.) \\ 2 Indian Health Outcomes, Public Health and Economics Research Center, L. V. Prasad Eye Institute, \\ Hyderabad 500034, India \\ 3 LVPEI Center for Innovation, L.V. Prasad Eye Institute, Banjara Hills, Hyderabad 500034, India; \\ nareenmolugu@gmail.com \\ * Correspondence: vipin@lvpei.org; Tel.: +040-68102367
}

Citation: Sai Prashanthi, G.; Molugu, N.; Kammari, P.; Vadapalli, R.; Das,

A.V. Forecast of Outpatient Visits to a Tertiary Eyecare Network in India Using the EyeSmart Electronic Medical Record System. Healthcare 2021, 9, 749. https://doi.org/ $10.3390 /$ healthcare 9060749

Academic Editor: Tin-Chih Toly Chen

Received: 28 May 2021

Accepted: 15 June 2021

Published: 18 June 2021

Publisher's Note: MDPI stays neutral with regard to jurisdictional claims in published maps and institutional affiliations.

Copyright: (c) 2021 by the authors. Licensee MDPI, Basel, Switzerland. This article is an open access article distributed under the terms and conditions of the Creative Commons Attribution (CC BY) license (https:/ / creativecommons.org/licenses/by/ $4.0 /)$.

\begin{abstract}
India is home to 1.3 billion people. The geography and the magnitude of the population present unique challenges in the delivery of healthcare services. The implementation of electronic health records and tools for conducting predictive modeling enables opportunities to explore time series data like patient inflow to the hospital. This study aims to analyze expected outpatient visits to the tertiary eyecare network in India using datasets from a domestically developed electronic medical record system (eyeSmart ${ }^{\mathrm{TM}}$ ) implemented across a large multitier ophthalmology network in India. Demographic information of 3,384,157 patient visits was obtained from eyeSmart EMR from August 2010 to December 2017 across the L.V. Prasad Eye Institute network. Age, gender, date of visit and time status of the patients were selected for analysis. The datapoints for each parameter from the patient visits were modeled using the seasonal autoregressive integrated moving average (SARIMA) modeling. SARIMA $(0,0,1)(0,1,7)_{7}$ provided the best fit for predicting total outpatient visits. This study describes the prediction method of forecasting outpatient visits to a large eyecare network in India. The results of our model hold the potential to be used to support the decisions of resource planning in the delivery of eyecare services to patients.
\end{abstract}

Keywords: forecasting; electronic health records; health resources; patient flow; SARIMA

\section{Introduction}

The geography and the magnitude of the population in India present unique challenges in the delivery of healthcare services. Digitization of patient records brings with it the benefits of real-time access to information and enables a better strategy for enhancing future care. Various studies have predicted patterns in patient care such as patient flow for outpatient consultation [1], discharge prioritization for inpatients [2], readmission rates in hospitals [3,4] and adverse drug reactions [5]. Ramos et al. [1] described a predictive model which calculated the patient flow based on geospatial variables in a medium-sized Spanish city. Patients who had a higher socioeconomic status and those in the age groups of 25-34 and 55-65 years paid visits to health centres less often. Monday and Friday were the days of the greatest and lowest demand, respectively. February had the highest influx of patients on a monthly basis. There were significantly more patient visits during days with poor air quality and high relative humidity. Barnes et al. [2] presented a model which used supervised machine learning methods to predict the total number of discharges in a day for an inpatient medical unit. McLaren et al. [3] described a model which predicted the readmission rates among heart failure patients based on their prior admission status. They observed that the risk of readmission significantly increases with the increase in the number of prior admissions after adjusting for the related clinical variables. Zhao et al. [5] 
proposed a predictive model to identify adverse events due to drugs and further presented solutions to few of the challenges it presented.

The use of statistical modeling to predict healthcare outcomes is breaking new ground in the field of biomedical informatics and data science. The implementation of electronic health records (EHR) and tools for conducting predictive modeling enables vast opportunities to explore time series data like patient inflow to the hospital. Historical traits are assumed to manifest themselves in the future in time series models [6]. Autoregressive integrated moving average (ARIMA) is a univariate stochastic model that uses probability and statistics for forecasting purposes. Seasonal ARIMA (SARIMA) modeling approach, a variant of ARIMA, is used in time series that have a tendency of showing a periodic behavior after certain time intervals [7]. Efficient management of eyecare services can be enhanced with predictive forecasts of future eyecare demand and resource availability in real time. In the present analysis using a SARIMA model, we aimed to evaluate the expected outpatient visits to a large tertiary eyecare network in India. We present a predictive model to forecast the presentation of patients that can assist in better resource allocation for an efficient delivery of eyecare services.

\section{Materials and Methods}

EyeSmart Electronic Medical Record (EMR) (eyeSmart ${ }^{\mathrm{TM}}$ ) is an electronic medical record system developed in-house at the L.V. Prasad Eye Institute, India. The project which started in August 2010 has successfully completed digitization of the entire LVPEI network which comprises centers which span four states of India. On the average, 4500 patients visit the network on a given day. The EMR system allows structured documentation of demographic and clinical information which are stored in the database of the respective centers. The study protocols and procedures adhered to the tenets of the declaration of Helsinki [8]. The data were made anonymous prior to access by the authors.

\section{Data Preparation}

Demographic information of 3,384,157 patient visits was obtained from eyeSmart EMR from August 2010 to December 2017 across the LVPEI network. Data of the four tertiary centers, namely, Hyderabad campus (KAR), Vishakhapatnam campus (GMRV), Bhubaneswar campus (MTC) and Vijayawada campus (KVC) were considered for analysis. The length of time series data of patient visits was 1250 days for the KAR, 1809 days for the GMRV, 1986 days for the MTC and 2793 days for the KVC. The parameters of age, gender, date of visit and time status of the patients were selected for analysis. The subset of patients $0-1$ years of age and all the patients who presented on time for the appointment were excluded from the analysis due to sparse data. The time status of the patient at presentation was defined as "before time" or "delayed" with respect to their time of appointment. The datapoints in each parameter from the patient visits were modeled using a train-test split, with a ratio of 80:20. This split was formed for the development and validation of the predictive model. The data for training and testing the model were selected from the KVC with 481,262 outpatient visits. The training and testing sets were separated by time. The training data consisted of 2241 days. The predictive model was tested by a one-week prediction of the parameters for 52 weeks. The model was trained on the data which were recorded before the date and validated on the data from a week ahead from the date.

\section{Results}

The following sequential steps were followed for SARIMA modeling and forecasting.

\subsection{Exploratory Data Analysis and Data Preprocessing}

\subsubsection{Data Visualization}

The daily outpatient visits show an overall increasing trend (Figure 1). A significant number of patients visited the center on most of the days except for few days like sundays where there was a dip in the total number of patients who visited the center. 


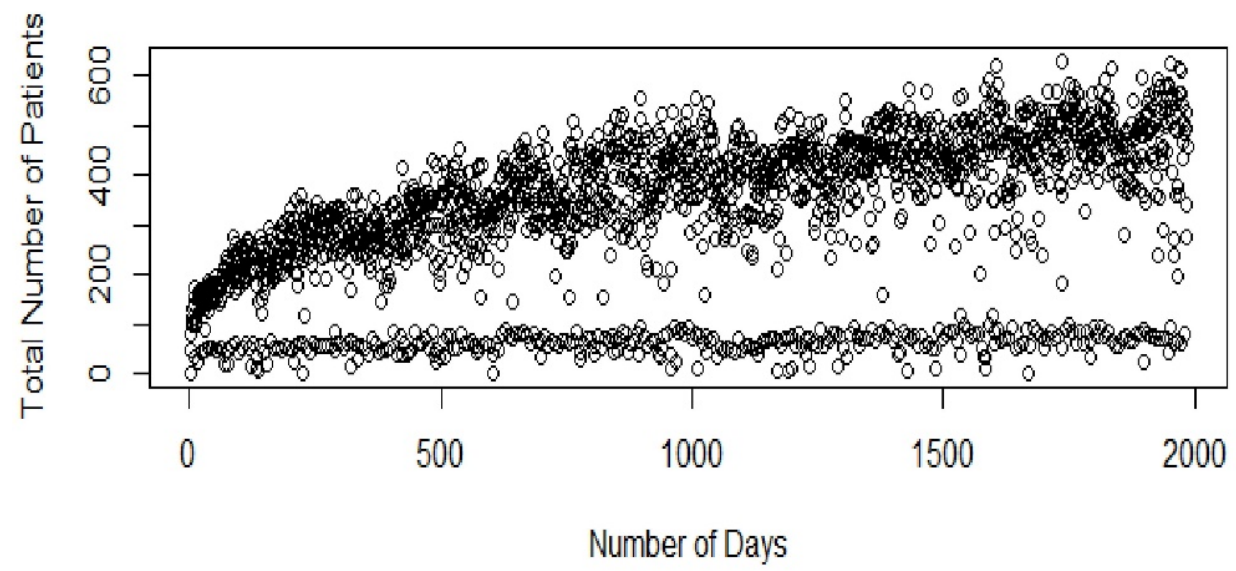

Figure 1. Total patient inflow (KVC).

The periodogram of the total number of patients suggests weekly seasonality (Figure 2). The periodogram shows spikes at frequencies of $0.14,0.28,0.42$, etc., confirming seasonality with a time period of seven days.

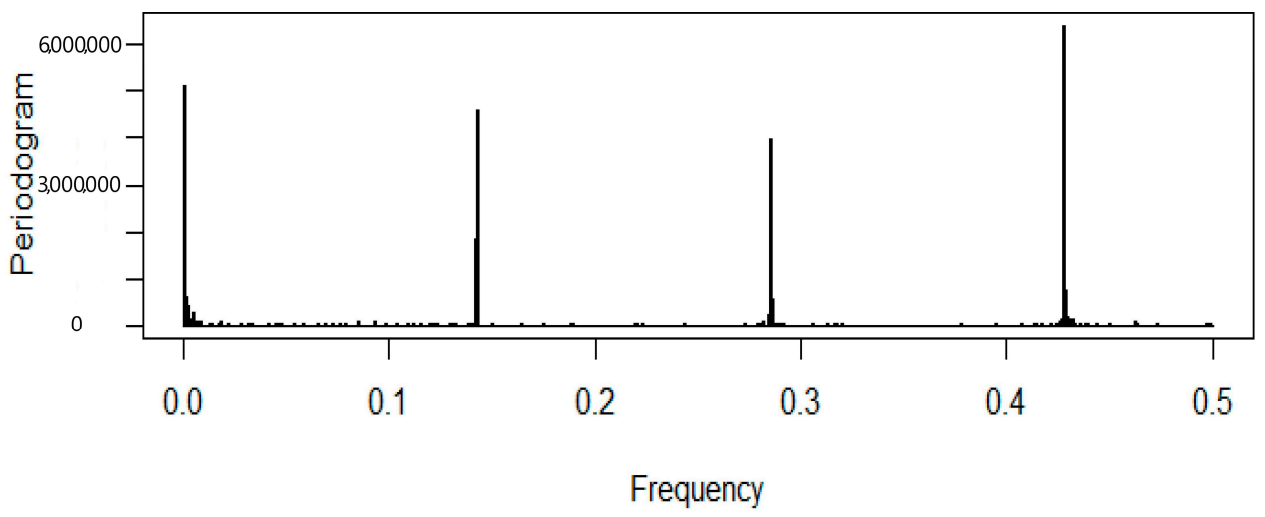

Figure 2. Periodogram of the total patients (KVC).

\subsubsection{Stationarity Check}

Visualization tools such as the sample autocorrelation function (ACF) and sample partial autocorrelation functions (PACF) were used to examine stationarity of the series (Figures 3 and 4). The Kwiatkowski-Philips-Schmidt-Shin (KPSS) test at the 0.05 significance level $(\alpha=0.05)$ was used to understand the type of non-stationarity in the data and suggested the necessary transformations. From the KPSS test, it was observed that the series which is differenced with the lag of 7 was trend-stationary. The autocorrelation plot (Figure 3) showed spikes at lag 7 and its multiples and the partial autocorrelation plot (Figure 4) demonstrated an initial significant spike with a rapid decline at multiples of $\operatorname{lag} 7$, both indicating a seasonal moving average term. Similar observations were in other categories as well; hence, similar procedures were followed to model other time-series data. 


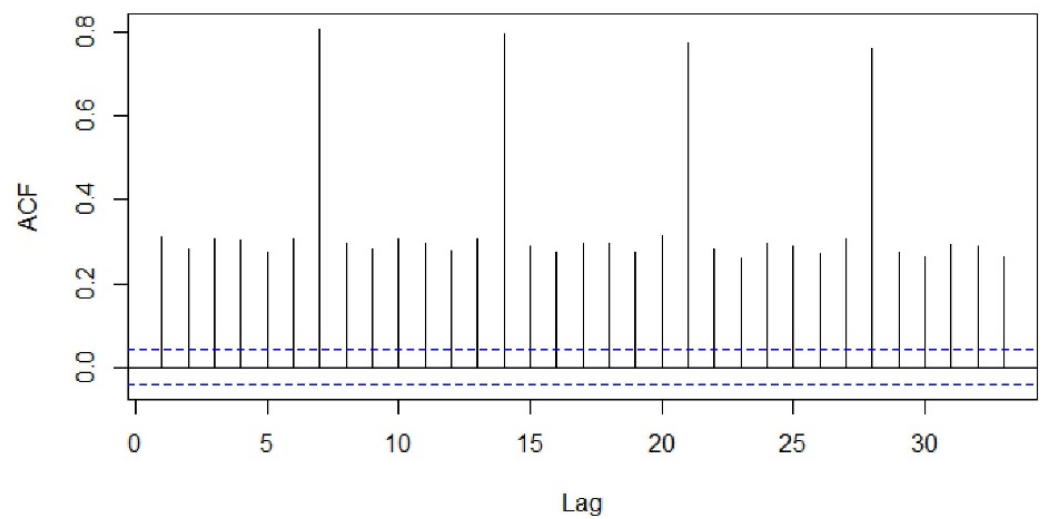

Figure 3. Autocorrelation function of the total number of patients.

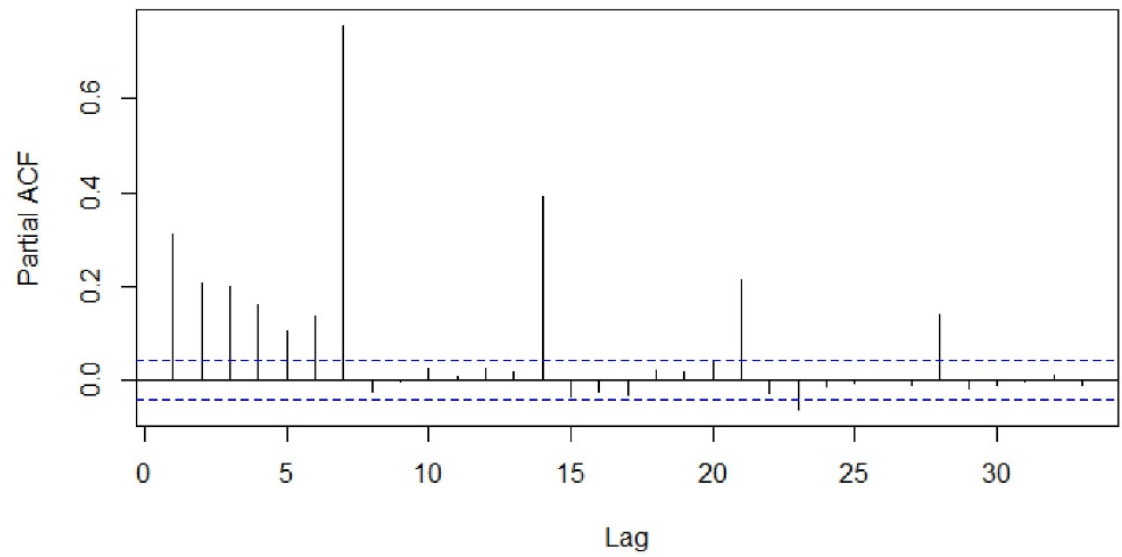

Figure 4. Partial autocorrelation function of the total number of patients.

\subsection{Model Identification and Diagnostic Checking}

The type (seasonal or non-seasonal) and $\operatorname{order}(\mathrm{p}, \mathrm{q}, \mathrm{P}, \mathrm{Q})$ of model parameters were identified on the basis of the ACF, PACF diagrams and Akaike information criterion (AIC) values. Different seasonal moving average (MA) models as suggested by the ACF and PACF plots were applied to our differenced data and the proximity of the model residuals to white noise was observed. The ACF and PACF of residuals of the ARIMA $(0,0,0)(0,1,7)_{7}$ applied to the time-series data were closest to the white noise (Figures 5 and 6). However, the ACF and PACF values observed at lag 1 suggested additional AR (autoregressive) or MA terms in our present model.

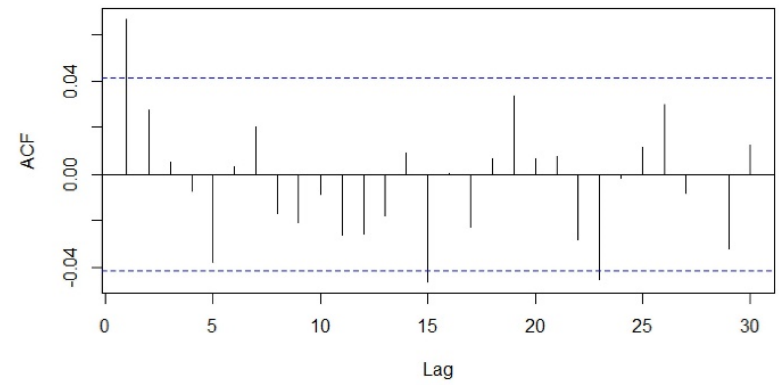

Figure 5. Autocorrelation function of residuals of ARIMA $(0,0,0)(0,1,7)$. 


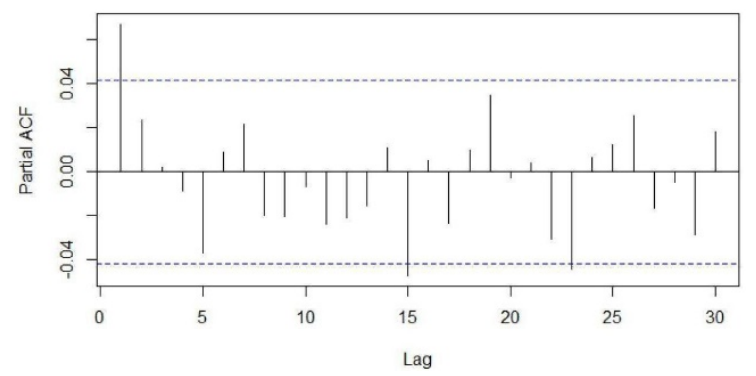

Figure 6. Partial autocorrelation function of residuals of ARIMA $(0,0,0)(0,1,7)$.

ARIMA $(0,0,1)(0,1,7)_{7}$ was found to be the best model for the total patient footfall data with an estimated sigma square of 1864, log likelihood of -11,548.23 and AIC value of 23,114.46. The majority of values of the ACF and PACF plots were within the confidence intervals which resemble the signature plots for white noise (Figures 7 and 8 ). The selection of the model for the respective parameters based on the AIC values and parsimony criteria is detailed in Table 1.

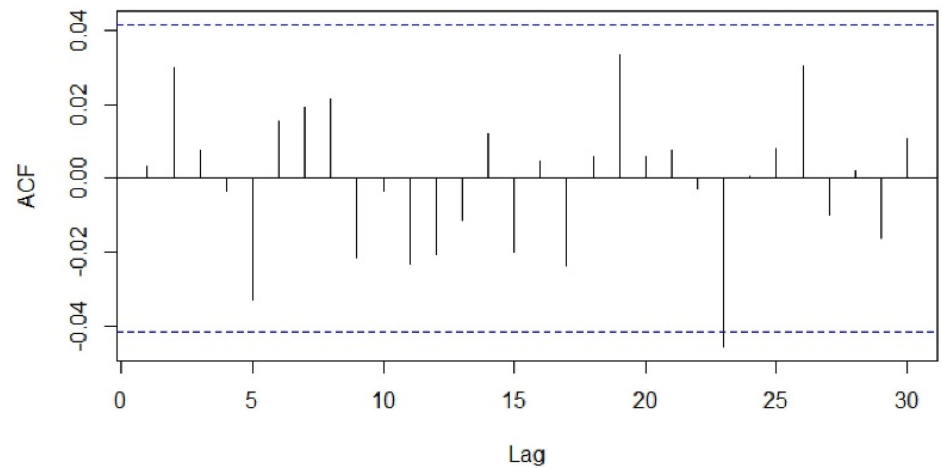

Figure 7. Autocorrelation function of residuals of ARIMA $(0,0,1)(0,1,7)$.

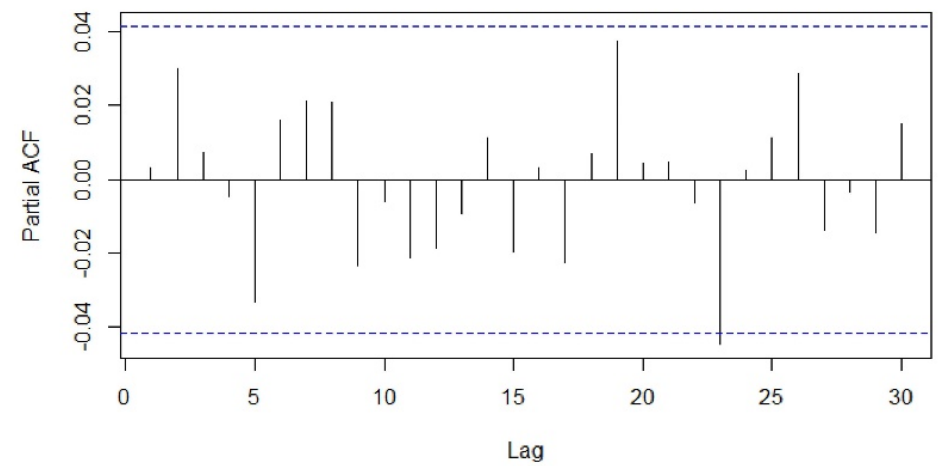

Figure 8. Partial autocorrelation function of residuals of ARIMA $(0,0,1)(0,1,7)$.

Table 1. AIC values of possible SARIMA models.

\begin{tabular}{ccccc}
\hline Parameter & $\mathbf{( 0 , 0 , 0 ) ( 0 , 1 , 7 ) _ { \mathbf { 7 } }}$ & $\mathbf{( 0 , 0 , 1 ) ( 0 , 1 , 7})_{\mathbf{7}}$ & $\mathbf{( 1 , 0 , 0 ) ( 0 , 1 , 7 ) _ { \mathbf { 7 } }}$ & $\mathbf{( 1 , 0 , 1 ) ( 0 , 1 , 7 ) _ { \mathbf { 7 } }}$ \\
\hline Male & $20,507.11$ & $20,506.7$ & $20,506.8$ & $20,508.45$ \\
Female & $20,002.47$ & $19,996.45$ & $19,996.72$ & $19,998.37$ \\
Pediatric & $16,727.46$ & $16,729.34$ & $16,729.33$ & $16,726.48$ \\
Adult & $20,513.51$ & $20,515.45$ & $20,515.46$ & $20,517.45$ \\
Elderly & $19,066.63$ & $19,051.54$ & $19,049.88$ & $19,049.98$ \\
Before time & $21,557.36$ & $21,556.42$ & $21,556.58$ & $21,558.15$ \\
Delayed & $19,089.98$ & $19,083.4$ & $19,083.14$ & $19,084.94$ \\
Total & $23,117.58$ & $23,114.46$ & $23,114.66$ & $23,116.3$ \\
\hline
\end{tabular}




\subsection{Evaluation Protocol}

Our training and testing sets were separated by time. The model was trained using data from the past and applied to the future data. The training data consisted of 1986 days from 2 June 2013 to 7 October 2017. The testing data consisted of 364 days from 8 October 2017 to 7 October 2018.

\subsection{Measuring Forecast Performance}

We compared the forecasts obtained using our proposed approach with the actual recorded values from the EMR on the measures of the root-mean-square error (RMSE), mean absolute error (MAE), mean absolute percentage error (MAPE) and symmetric mean absolute percentage error (SMAPE).

The modeling process found the following SARIMA, $(0,0,1)(0,1,7)_{7}$, provided the best fit for predicting total outpatient visits, generating the median of $37.56,48.01$ and $16.82 \%$ for MAE, RMSE, MAPE, respectively, in the validation dataset. Table 2 shows median error values of the predictive model.

Table 2. Median error values of the predictive model.

\begin{tabular}{cccc}
\hline Parameter & Mean Absolute Error & $\begin{array}{c}\text { Root-Mean-Square } \\
\text { Error }\end{array}$ & $\begin{array}{c}\text { Mean Absolute } \\
\text { Percentage Error }\end{array}$ \\
\hline Male & 22.98 & 28.36 & $22.99 \%$ \\
Female & 17.53 & 24.58 & $17.03 \%$ \\
Pediatric & 11.84 & 15.07 & $62.75 \%$ \\
Adult & 19.97 & 26.62 & $18.36 \%$ \\
Elderly & 16.97 & 21.91 & $23.79 \%$ \\
Before time & 30.16 & 36.72 & $44.17 \%$ \\
Delayed & 22.36 & 27.10 & $18.56 \%$ \\
Total & 37.56 & 48.01 & $16.82 \%$ \\
\hline
\end{tabular}

Different SARIMA models (Table 1) were used to make predictions of total outpatient visits and associated parameters for the week ahead. The prediction model was run for 52 weeks, spanning an entire year from 6 May 2017 to 6 May 2018. We used the SMAPE as the performance metric to account for the days where the actual value was zero. The comparison of total outpatient visits between the actual and predicted values for the KVC campus is shown in Figure 9. The number of weeks where the prediction was within the $20 \%$ margin of the SMAPE is detailed in Table 3.

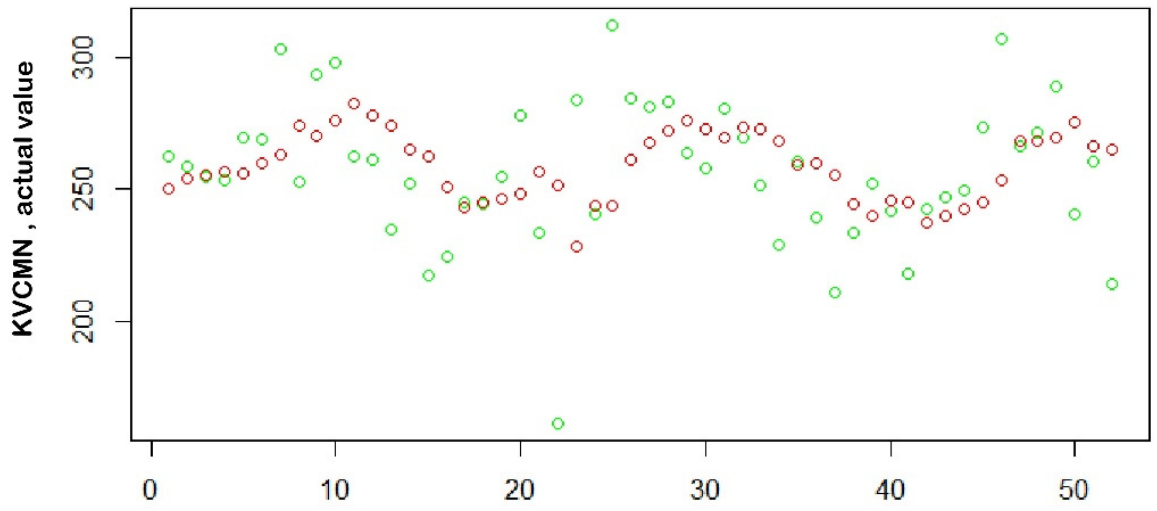

Figure 9. Comparison of actual (green) versus predicted (red) values. 
Table 3. Performance measurement of the model at 52 weeks (KVC).

\begin{tabular}{cc}
\hline Parameter & No. of Weeks (Error < 20\%) \\
\hline Male & $51(98.07 \%)$ \\
Female & $50(96.15 \%)$ \\
Delayed & $51(98.07 \%)$ \\
Before time & $44(84.61 \%)$ \\
Pediatric & $37(71.15 \%)$ \\
Adult & $51(98.07 \%)$ \\
Elderly & $51(98.07 \%)$ \\
Total & $51(98.07 \%)$ \\
\hline
\end{tabular}

Figure 10 shows the prediction of the total patient footfall for the next seven days; an option to visualize a one-week-ahead forecast of variables such as the total count, male count, female count, pediatric count, adult count, delayed patient count and count of patients who are before the appointed time is given in the dropdown list. As a part of the ongoing validation of the model with the actual footfalls in the hospital, actual vs. predicted values of the previous seven days were showcased to assess the accuracy of the prediction. The forecasting model was integrated into the eyeSmart EMR system for the administrative staff.

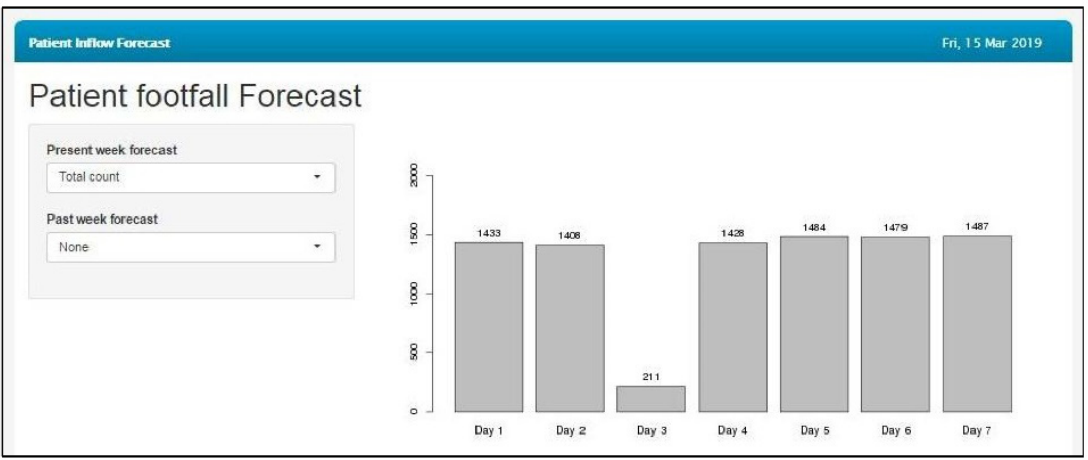

Figure 10. Prediction of the total patient count for the next seven days.

\section{Discussion}

\subsection{Principal Results}

This study described the methodology of predicting outpatient visits in a large eyecare network in India. The parameters of age, gender and time status at presentation were also predicted to gain better insights to provide efficient eyecare services. The modeling process found SARIMA $(0,0,1)(0,1,7)_{7}$ to provide the best fit for forecasting total outpatient inflow. The MAPE of $16.82 \%$ was observed for the predictive model which forecasted the total outpatient inflow.

\subsection{Comparison with Prior Works}

Ramos et al. [1] predicted the expected outpatient visits to primary health centers in a medium-sized city in Spain. The study used multiple regression analysis and showed an improvement in error values on the addition of geospatial variables such as temperature, air quality, relative humidity and economic status. Further, Luo et al. [9] described a model which was used for forecasting outpatient visits daily about a week ahead from the data which contained one year of daily visits of outpatients to a large hospital in Chengudu. The combinatorial model which combined SARIMA and simple exponential smoothening (SES) was used in this study. The MAPE values of only the SARIMA model for respiratory and endocrine outpatient visits was $15.26 \%$ and $11.77 \%$, respectively for a seven-day forecast horizon. In addition, Calegari et al. [10] forecasted daily visits to a tertiary care teaching hospital in Brazil using different mathematical models. Model accuracy was evaluated 
using the MAPE. Their MAPE at seven days was comparable, at 12.01\%, using the SARIMA model. Huang et al. [11] used a hybrid prediction model which combined ARIMA and filtering to accurately forecast the demand for medical services in the medium as well as short term. Another retrospective study in a medical center in Taiwan forecasted emergency visits using time series analysis. They identified that ARIMA $(0,0,1)$ was the best fit and yielded the MAPE of $8.91 \%$ [12]. A group in China conducted an observational study which analyzed patient's visits across 10 years at an eyecare facility [13]. They observed a continuous increase in patient visits over the years leading to an increased demand and hence more burden on the eyecare system.

Numerous studies have been undertaken to forecast new admission inpatients, outpatient visits and visits to blood sampling rooms and emergency departments [14-18]. Predictive models can be used to support the decisions regarding the allocation of resources and can significantly reduce the burden on healthcare. In a country of 1.3 billion, data science holds a potential for predictive models to be applied to healthcare delivery. To the best of our knowledge, there is no study currently in literature describing the use of data modeling in predicting outpatient visits in eyecare in India.

\subsection{Limitations}

The model used in this study is used only for short-term forecasting. Apart from the time series data used for prediction, many other influence factors such as preference of a particular doctor by the patient and supply of outpatient resource will also have a significant impact on the visits of the patients. The model can be made more efficient by taking these features into consideration.

\section{Conclusions}

In conclusion, this study contributes to the exploration of the prediction method to forecast outpatient visits in India's largest eyecare centers. Our study confirms that the daily patient inflow follows seasonal weekly patterns. Further, it shows that SARIMA models can be implemented to accurately predict forecasts of highly complex patient inflow data, mainly in the short term. This model is feasible in terms of implementation and computation of prediction for a week ahead, yielding small mean (0.02) and lower standard deviation (17.34) of the residuals. The current prediction model is integrated into the eyeSmart EMR system across the four tertiary centers in the LVPEI network to validate outpatient visits predictions in the real-life scenario. The results of our model hold a potential to be used to support the decisions of resource planning in the delivery of eyecare services to the patients.

Future work mainly involves building models which can forecast outpatient visits in the middle and long term. The inclusion of geospatial variables like temperature, air quality and relative humidity can also increase the prediction accuracy of the model. Our future work will involve addition of these variables to the model to increase prediction accuracy.

Author Contributions: Conceptualization, N.M. and A.V.D.; methodology, N.M. and A.V.D.; software, R.V. and P.K.; validation, N.M. and A.V.D.; formal analysis, N.M.; investigation, A.V.D.; resources, A.V.D. and R.V.; data curation, N.M., A.V.D. and R.V.; writing-original draft preparation, N.M., A.V.D. and P.K.; writing-review and editing, G.S.P., N.M., A.V.D. and P.K.; visualization, N.M.; supervision, A.V.D.; project administration, A.V.D. All authors have read and agreed to the published version of the manuscript.

Funding: This research was funded by Hyderabad Eye Research Foundation, India.

Institutional Review Board Statement: The study was conducted according to the guidelines of the Declaration of Helsinki.

Informed Consent Statement: Informed consent was obtained from all subjects involved in the study.

Data Availability Statement: Data sharing not applicable. 
Acknowledgments: The authors wish to acknowledge the support of our Department of eyeSmart EMR and the AEye team, specifically, Mohammad Pasha.

Conflicts of Interest: The funders had no role in the design of the study; in the collection, analyses, or interpretation of data; in the writing of the manuscript, or in the decision to publish the results.

\section{References}

1. Ramos, I.; Cubillas, J.J.; Feito, F.R.; Ureña, T. Spatial analysis and prediction of the flow of patients to public health centres in a middle-sized Spanish city. Geospat. Health 2016, 11. [CrossRef] [PubMed]

2. Barnes, S.; Hamrock, E.; Toerper, M.; Siddiqui, S.; Levin, S. Real-time prediction of inpatient length of stay for discharge prioritization. JAMA 2016, 23, e2-e10. [CrossRef] [PubMed]

3. McLaren, D.P.; Jones, R.; Plotnik, R.; Zareba, W.; McIntosh, S.; Alexis, J.; Chen, L.; Block, R.; Lowenstein, C.J.; Kutyifa, V. Prior hospital admission predicts thirty-day hospital readmission for heart failure patients. Cardiol. J. 2016, 23, 155-162. [CrossRef] [PubMed]

4. Chen, R.; Su, H.; Khalilia, M.; Lin, S.; Peng, Y.; Davis, T.; Hirsh, D.A.; Searles, E.; Tejedor-Sojo, J.; Thompson, M.; et al. Cloud-based Predictive Modeling System and its Application to Asthma Readmission Prediction. AMIA Annu. Symp. Proc. $2015,5,406-415$.

5. Zhao, J.; Henriksson, A.; Asker, L.; Boström, H. Predictive modeling of structured electronic health records for adverse drug event detection. BMC Med. Inform. Decis. Mak. 2015, 4, 1472-6947. [CrossRef] [PubMed]

6. Vahdat, S.F.; Sarraf, A.; Shamsnia, A.; Shahidi, N. Prediction of monthly mean Inflow to Dez Dam reservoir using time series models (Box-jenkins). In Proceedings of the International Conference on Environment and Industrial Innovation, Sinagpore, 4-5 June 2011; Volume 12, pp. 162-166.

7. Box, G.E.; Jenkins, G.M.; Reinsel, G.C.; Ljung, G.M. Time Series Analysis, Forecasting and Control, 4th ed.; Wiley \& Sons: Hoboken, NJ, USA, 2008.

8. Association, W.M. World Medical Association Declaration of Helsinki: Ethical principles for medical research involving human subjects. JAMA 2013, 310, 2191-2194. [CrossRef]

9. Luo, L.; Luo, L.; Zhang, X.; He, X. Hospital daily outpatient visits forecasting using a combinatorial model based on ARIMA and SES models. BMC Health Serv. Res. 2017, 17, 469. [CrossRef] [PubMed]

10. Calegari, R.; Fogliatto, F.S.; Lucini, F.R.; Neyeloff, J.; Kuchenbecker, R.S.; Schaan, B.D. Forecasting Daily Volume and Acuity of Patients in the Emergency Department. Comput. Math. Methods Med. 2016, 2016, 3863268. [CrossRef] [PubMed]

11. Huang, Y.; Xu, C.; Ji, M.; Xiang, W.; He, D. Medical service demand forecasting using a hybrid model based on ARIMA and self-adaptive filtering method. BMC Med. Inf. Decis. Mak. 2020, 20, 237. [CrossRef] [PubMed]

12. Juang, W.C.; Huang, S.J.; Huang, F.D.; Cheng, P.W.; Wann, S.R. Application of time series analysis in modelling and forecasting emergency department visits in a medical centre in Southern Taiwan. BMJ Open 2017, 7, e018628. [CrossRef]

13. Yuan, M.; Chen, W.; Wang, T.; Song, Y.; Zhu, Y.; Chen, C.; Yang, Y.; Liu, Y.; Li, Y.; Lin, H. Exploring the growth patterns of medical demand for eye care: A longitudinal hospital-level study over 10 years in China. Ann. Transl. Med. 2020, 8, 1374. [CrossRef]

14. Huang, D.; Wu, Z. Forecasting outpatient visits using empirical mode decomposition coupled with back-propagation artificial neural networks optimized by particle swarm optimization. PLoS ONE 2017, 12, e0172539. [CrossRef] [PubMed]

15. Kim, K.-R.; Park, J.-E.; Jang, I.-T. Outpatient forecasting model in spine hospital using ARIMA and SARIMA methods. J. Hosp. Manag. Health Policy 2020, 2020, 4. [CrossRef]

16. Sumitra, I.D.; Basri, I. Forecasting the Number of Outpatient Patient Visits Using the ARIMA, SES And Holt-Winters Methods at XYZ Community Health Center. IOP Conf. Ser. Mater. Sci. Eng. 2020, 879, 012060. [CrossRef]

17. Zhang, X.; Yu, Y.; Xiong, F.; Luo, L. Prediction of Daily Blood Sampling Room Visits Based on ARIMA and SES Model. Comput. Math. Methods Med. 2020, 2020, 1720134. [CrossRef] [PubMed]

18. Zhou, L.; Zhao, P.; Wu, D.; Cheng, C.; Huang, H. Time series model for forecasting the number of new admission inpatients. BMC Med. Inf. Decis. Mak. 2018, 18, 39. [CrossRef] [PubMed] 